\title{
Corrigendum
}

\section{Applying feminist, multicultural, and social justice theory to diverse women who function as caregivers in end-of-life and palliative home care}

CHRISTOPHER J. MACKINNON, B.A., M.A.

Department of Educational and Counselling Psychology, McGill University, Montreal, Québec, Canada; Palliative Care Research, S.M.B.D. Jewish General Hospital, Montreal, Quebéc, Canada; Palliative Care Unit, Montreal General Hospital, Montreal, Quebéc, Canada

(Volume 7, Number 4, 2009; Pages 501-512)

\begin{abstract}
Objective: Women are largely responsible for providing care to terminally ill family members at home. The goal of this review is to conceptualize diverse women's experiences in palliative home care from feminist, multicultural, and social justice perspectives.

Methods: Peer-reviewed manuscripts were identified using the following databases: CINAHL, psycINFO, and pubMED. The following search terms were used: women/mothers/daughters, Caregiving family caregivers, feminism, culture, multiculturalism, and palliative home care. Article reference lists were also reviewed. The majority of pertinent articles which formed the basis for the arguments presented were drawn from nursing, medicine, and counseling psychology schlorship.

Results: The application of feminist, multicultural, and social justice theroy brings to attention several potential issues female caregivers may experience. First, there exist diverse ways in which women's caregiving is manifested that tend to correspond with variations in culture, relationship, and age. Second, it is important to attend to changing expectations placed on women as a result of caregiving at the end of life. Third, the changing power dynamics women may experience in end of life caregiving are very complex.

Significance of results: The principle finding of the review was the highlighting of potential risks that culturally diverse female caregivers are likely to face at the end of life. The application of social justice theory provides a number of implications for practice and policy. Specifically, by identifying significant concerns regarding female caregivers in palliative home care, as well as suggesting ways to appropriately attend to these concerns, any oppression of women is less likely to be perpetuated. Specific areas for future research in this domain are identified.
\end{abstract}

